ESAIM: PROCEEDINGS AND SURVEYS, October 2015, Vol. 51, p. 74-88

A. Garivier et al, Editors

e-mail: quentin. berger@upmc.fr

\title{
INFLUENCE OF DISORDER FOR THE POLYMER PINNING MODEL
}

\author{
Quentin Berger ${ }^{1}$
}

\begin{abstract}
When studying physical systems, the influence of disorder on the phase transition is a central question: one wants to determine whether an arbitrary small amount of randomness modifies the critical properties of the system, with respect to the non-disordered case. We present here an overview of the mathematical results obtained to answer that question in the context of the polymer pinning model. In the IID case, the picture of disorder relevance/irrelevance is by now established, and follows the so-called Harris criterion: disorder is irrelevant if $\nu^{\text {hom }}>2$ and relevant if $\nu^{\text {hom }}<2$, where $\nu^{\text {hom }}$ is the order of the homogeneous phase transition. The marginal case $\nu^{\text {hom }}=2$ has been subject to controversy in the physics literature in the context of pinning models, but has recently been fully settled. In the correlated case, Weinrib and Halperin predicted that, if the two-point correlations decay as a power law with exponent $\xi>0$, then the Harris criterion would be modified if $\xi<1$ : disorder should be relevant whenever $\nu^{\text {hom }}<2 \max (1,1 / \xi)$. It turns out that this prediction is not accurate: the key quantity is not the decay exponent $\xi$, but the occurrence of rare regions with atypical disorder. An infinite disorder regime may appear, in which the relevance/irrelevance picture is crucially modified. We also mention another recent approach to the question of the influence of disorder for the pinning model: the persistence of disorder when taking the scaling limit of the system.
\end{abstract}

\section{INTRODUCTION AND PHYSICAL MOTIVATIONS}

The question of disorder relevance for a physical system has been a central question for a century, and before turning to the specific case of the pinning model, we introduce it in a general context.

\section{The Harris criterion for general disordered systems}

Consider a physical system on $\mathbb{Z}^{d}$ to which is associated a parameter $h \in \mathbb{R}$ homogeneous over space, and suppose that the system is undergoing a phase transition at some critical parameter $h_{c}$. This is referred to as the pure or homogeneous model, and one might think of the Ising model, percolation, or the pinning (or wetting) model. One then desires to know whether the introduction of a small quantity of randomness (or inhomogeneities, or impurities) modifies the characteristics of the phase transition. More precisely, let $\omega=\left(\omega_{x}\right)_{x \in \mathbb{Z}^{d}}$ be a stationary and ergodic field of random variables, with $\mathbb{E}\left[\omega_{x}\right]=0$ and $\mathbb{E}\left[\left(\omega_{x}\right)^{2}\right]=1$ (for simplicity): $\omega$ is referred to as randomness or disorder. Then, for a parameter $\beta>0$ (the intensity of disorder), one modifies locally the parameter $h$ in the system, replacing it by $h+\beta \omega_{x}$ at site $x \in \mathbb{Z}^{d}$ : one obtains a disordered system. The question are then numerous: does the phase transition survive? what can one say about the disordered critical parameter $h_{c}(\beta, \omega)$ ? are the characteristics of the disordered phase transition similar to that of the homogeneous one?

This last issue has been the center of much attention: if for an arbitrarily small amount of randomness (that is for any arbitrarily small $\beta>0$ ) the properties of the disordered and homogeneous phase transition are different, then disorder is said to be relevant. Otherwise, it is said to be irrelevant.

${ }^{1}$ LPMA, Université Pierre et Marie Curie, Case courrier 188 - 4 Place Jussieu, 75252 Paris Cedex 05 - France

(C) EDP Sciences, SMAI 2015 
In his seminal paper, Harris [32] explains that disorder relevance can be decided only through the order $\nu^{\text {hom }}$ of the homogeneous phase transition: the correlation length of the homogeneous system diverges as $\left|h-h_{c}\right|^{-\nu^{\text {hom }}+o(1)}$ as $h$ approaches $h_{c}$ (a precise definition of the order of the phase transition is given in the context of pinning model, see (5)). Harris predicts that an IID disorder is relevant if $\nu^{\text {hom }}<2 / d$ and irrelevant if $\nu^{\text {hom }}>2 / d$. The case $\nu^{\text {hom }}=2 / d$ is said to be marginal and needs to be specifically investigated for each model.

In the case of a correlated disorder, with two-point correlations decaying as a power $\xi$ of the distance, $|i-j|^{-\xi}$, Weinrib and Halperin [43] predict that the Harris criterion is not changed if $\xi>d$ (disorder is relevant is $\nu^{\text {hom }}<2 / d$ ), and modified if $\xi<d$ (disorder is relevant if $\nu^{\text {hom }}<2 / \xi$ ).

\section{Scope of the paper}

We focus here on the polymer pinning model, which was introduced by Poland and Scheraga [37] to describe the DNA denaturation phenomenon, but has also been used in other contexts: it has for example been employed as an effective model for the wetting of an interface on a disordered substrate (see [25,27] and references therein for an overview of the physical motivations). The model considers a directed polymer interacting with a defect line: one wants to describe its localization phase transition, and determine the influence of randomness on its characteristics. From its definition in Section 1, the pinning model is in turn a family of 1-dimensional models (interactions are concentrated on a 1-dimensional defect line), which spans the whole range of possible orders $\nu^{\text {pur }} \in[1+\infty]$ for the homogeneous phase transition (see Theorem 1.3). It is therefore a natural candidate to test the Harris prediction and also to investigate the marginal case.

Since this model is only 1-dimensional, Harris' prediction tells that disorder should be relevant if $\nu^{\text {hom }}<2$, and irrelevant if $\nu^{\text {hom }}>2$. Also, according to Weinrib and Halperin, this criterion should be preserved if $\xi>1$ and modified if $\xi<1$, where $\xi$ is the decay exponent of two-point correlations.

- In Section 1, we introduce the disordered pinning model and give its first properties.

- In Section 2, we review the existing results in the IID case, where the Harris criterion has been proven, and a simple necessary and sufficient condition for disorder relevance has been given recently [12] in the marginal case $\nu^{\text {hom }}=2$. We stress the influence of disorder fluctuations, and in particular the role of rare atypical regions in disorder relevance, see Section 2.2.

- In Section 3, we turn to a correlated environment, which has been the author's focus and main contribution the past few years. The Weinrib-Halperin's predictions have been found not to be valid in general: the important quantity to consider is not the two-point correlations decay exponent, but the exponential or sub-exponential decay of the size of rare favorable regions.

- Finally, we conclude by mentioning another related question in Section 4, which is a priori not that of disorder relevance as usually discussed, but brings a new point of view: the study of the scaling limit of the system, and the persistence of disorder in the continuum limit.

\section{The DISORDERED PINNING MODEL}

\subsection{Definition of the model}

The polymer. To model a polymer chain, one may use the graph of a directed random walk $\left(n, S_{n}\right)_{n \in \mathbb{N}}$ where $S$ is a random walk with IID increments on $\mathbb{Z}^{r}$. However, since we are only interested in the interactions with the defect line $\mathbb{N} \times\{0\}$, we simply need to consider the set $\tau$ of return times of $S_{n}$ to 0 (which is a 1-dimensional object), see Figure 1.

We therefore consider a slightly more general (1-dimensional) model. Let $\tau:=\left\{\tau_{0}=0, \tau_{1}, \ldots\right\}$ be a renewal process, whose law is denoted by $\mathbf{P}: \tau_{0}:=0$ and $\left(\tau_{i}-\tau_{i-1}\right)_{i \in \mathbb{N}}$ is a sequence of IID, $\mathbb{N}$-valued, random variables, with common inter-arrival distribution $\mathrm{K}(n):=\mathbf{P}\left(\tau_{1}=n\right)$. The set $\tau$ (with a slight abuse of notations) is interpreted as the set of contact points of the polymer with the defect line. We denote $\{n \in \tau\}$ the event that $n$ is a renewal point, that is that there exists some $k \in \mathbb{N}$ such that $\tau_{k}=n$. 


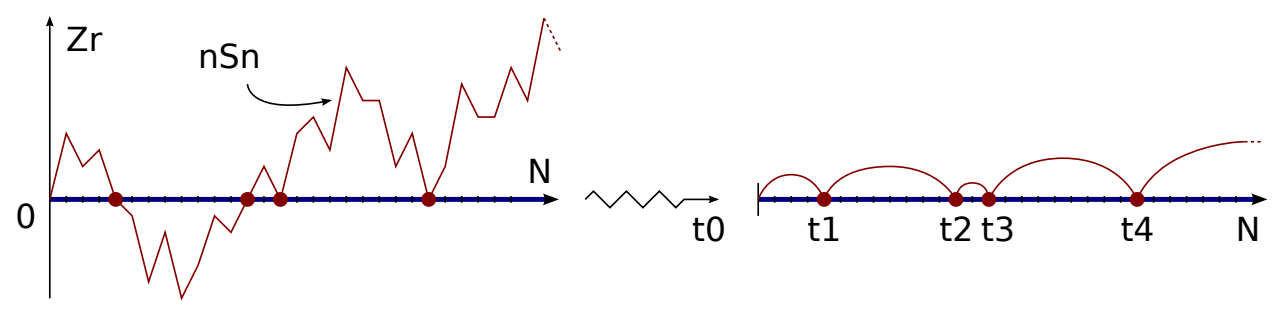

FIGURE 1. Polymer modeled by a directed random walk $\left(n, S_{n}\right)_{n \in \mathbb{N}}$ interacting with the defect line $\mathbb{N} \times\{0\}$, and its reduction to a renewal process. The defect line is 1-dimensional, and we are only interested in the sequence of contact points $\tau_{0}=0, \tau_{1}, \tau_{2}, \ldots$ represented here by dots.

One is lead to make the following assumption on the inter-arrival distribution:

Assumption 1.1. There exists a positive slowly-varying function $\varphi(\cdot)$ and some $\alpha \geq 0$ such that

$$
\mathbf{P}\left(\tau_{1}=n\right)=: \mathrm{K}(n) \stackrel{n \rightarrow \infty}{\sim} \varphi(n) n^{-(1+\alpha)} .
$$

This assumption is very natural. Indeed, if $S$ is a symmetric simple random walk on $\mathbb{Z}^{r}$, the first hitting time of $0, \tau_{1}$, is known to satisfy Assumption 1.1 (aside from periodicity considerations easily overcome by only considering even times of return): there are explicit constants $c_{r}$, such that, for $r=1, \alpha=1 / 2$ and $\lim _{n \rightarrow \infty} \varphi(n)=c_{1}$; for $r=2, \alpha=0$ and $\varphi(n) \stackrel{n \rightarrow \infty}{\sim} c_{2}(\log n)^{-2}$; and for $r \geq 3, \alpha=\frac{r}{2}-1$ and $\lim _{n \rightarrow \infty} \varphi(n)=c_{r}$. Assumption 1.1 also covers the case of a random walk on $\mathbb{Z}$ in the domain of attraction of a stable law, and of Bessel-type random walks on $\mathbb{Z}$, which can give asymptotically any $\alpha \geq 0$ and $\varphi(\cdot)$, see [2].

The random composition of the defect line. Let $\omega=\left(\omega_{n}\right)_{n \in \mathbb{N}}$ be a stationary ergodic sequence of random variables, whose law is denoted by $\mathbb{P}$. We assume that $\mathbb{E}\left[\omega_{1}\right]=0$ and $\mathbb{E}\left[\omega_{1}^{2}\right]=1$ for simplicity. We refer to $\omega$ as the disorder: it models the inhomogeneities of the defect line.

The polymer measure. Given a fixed realization of $\omega$ (quenched disorder), and parameters $\beta \geq 0$ (intensity of disorder) $h \in \mathbb{R}$ (homogeneous interaction), we define the measures $\mathbf{P}_{N, h}^{\beta, \omega}$ on trajectories of $\tau$ which are pinned at site $N$, as a Gibbs transformation of $\mathbf{P}$ :

$$
\frac{\mathrm{d} \mathbf{P}_{N, h}^{\beta, \omega}}{\mathrm{d} \mathbf{P}}(\tau):=\frac{1}{Z_{N, h}^{\beta, \omega}} \exp \left(\sum_{n=1}^{N}\left(\beta \omega_{n}+h\right) \mathbf{1}_{\{n \in \tau\}}\right) \mathbf{1}_{\{N \in \tau\}},
$$

where $Z_{N, h}^{\beta, \omega}$ is the partition function of the system, used to normalize $\mathbf{P}_{N, h}^{\beta, \omega}$ to a probability,

$$
Z_{N, h}^{\beta, \omega}=\mathbf{E}\left[\exp \left(\sum_{n=1}^{N}\left(\beta \omega_{n}+h\right) \mathbf{1}_{\{n \in \tau\}}\right) \mathbf{1}_{\{N \in \tau\}}\right] .
$$

The measure $\mathbf{P}_{N, h}^{\beta, \omega}$ corresponds to giving a reward (or a penalty, depending on its sign) $\beta \omega_{n}+h$ if there is a contact with the defect line at site $n$. The goal is then to study the properties of trajectories of $\tau$ under $\mathbf{P}_{N, h}^{\beta, \omega}$ as $N$ goes to infinity, for typical realizations of $\omega$, and how they depend on the parameters $\beta \geq 0, h \in \mathbb{R}$.

\subsection{The free energy and the pinning phase transition}

A central physical quantity is the free energy, or energy per monomer, which encodes many properties of the system. 
Proposition 1.2 (see [25], Ch. 4). The following limit exists $\mathbb{P}$-a.s. and in $\mathrm{L}^{1}$,

$$
\mathrm{F}(\beta, h):=\lim _{N \rightarrow \infty} \frac{1}{N} \log Z_{N, h}^{\beta, \omega}=\lim _{N \rightarrow \infty} \frac{1}{N} \mathbb{E} \log Z_{N, h}^{\beta, \omega}
$$

The quantity $\mathrm{F}(\beta, h)$ is called the quenched free energy of the system, and the map $(\beta, h) \mapsto \mathrm{F}(\beta, h)$ is nonnegative, convex, and non-decreasing in both variables.

With this proposition in hand, one observes a phase transition when making $h$ vary: there exists a quenched critical point $h_{c}^{\text {que }}(\beta)$ such that $\mathrm{F}(\beta, h)>0$ if and only if $h>h_{c}(\beta) ; h_{c}(\beta)=\inf \{h ; \mathrm{F}(\beta, h)>0\}=$ $\sup \{h ; \mathrm{F}(\beta, h)=0\}$. How to actually interpret this phase transition may still be unclear, but one can derive some path properties from the free energy, and relate the positivity of the free energy to localization properties. Indeed, a straightforward computation yields that $\partial_{h} \log Z_{N, h}^{\beta, \omega}=\mathbf{E}_{N, h}^{\beta, \omega}\left[\sum_{n=1}^{N} \mathbf{1}_{\{n \in \tau\}}\right]$. Therefore, using the convexity of F (and Proposition 1.2), we get that

$$
\partial_{h} \mathrm{~F}(\beta, h)=\lim _{N \rightarrow \infty} \mathbf{E}_{N, h}^{\beta, \omega}\left[\frac{1}{N} \sum_{n=1}^{N} \mathbf{1}_{\{n \in \tau\}}\right], \quad \mathbb{P}-a . s .
$$

when the derivative $\partial_{h} \mathrm{~F}(\beta, h)$ exists (it is however known that $h \mapsto \mathrm{F}(\beta, h)$ is $\mathrm{C}^{\infty}$ except possibly at $h_{c}^{\text {que }}(\beta)$ ).

The critical point therefore marks the transition between a localized phase $\mathcal{L}=\{(\beta, h) ; \mathrm{F}(\beta, h)>0\}=\{h>$ $\left.h_{c}^{\text {que }}(\beta)\right\}$, where there is (asymptotically) a positive density of contacts under $\mathbf{P}_{N, h}^{\beta, \omega}$, the polymer is pinned to the defect line; and a delocalized phase $\mathcal{D}=\{(\beta, h) ; \mathrm{F}(\beta, h)=0\}=\left\{h \leq h_{c}^{\text {que }}(\beta)\right\}$, where the density of contacts is (asymptotically) null, the polymer wandering away from the line. We give an overview of the phase diagram in Figure 2, with a sketch of typical trajectories in the localized and delocalized phase.

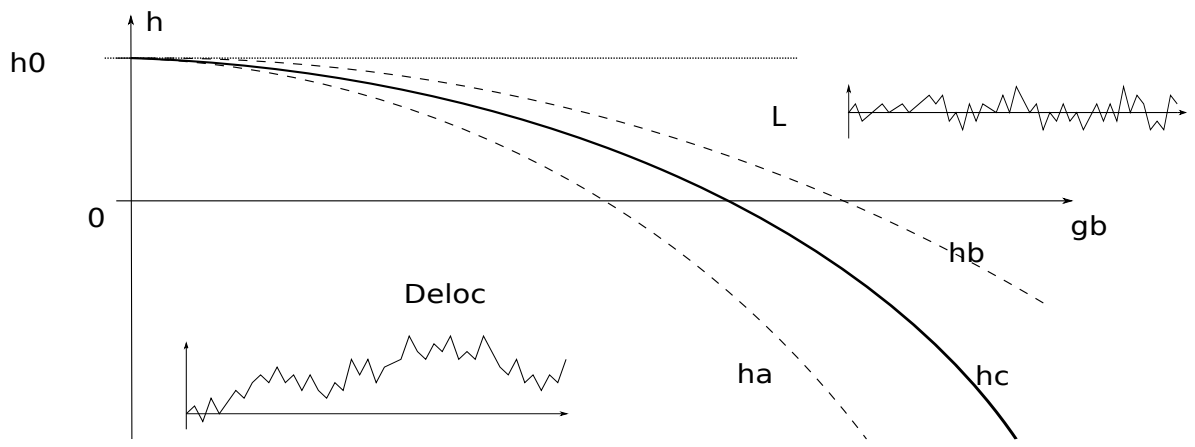

FIGURE 2. Phase diagram and typical trajectories in the localized and delocalized phases. Since $(\beta, h) \mapsto$ $\mathrm{F}(\beta, h)$ is convex, the region $\mathcal{D}$ is convex, so that $h_{c}(\beta)$ is a concave function of $\beta$.

Moreover, various bounds are known on the critical curve, giving some more information on the phase diagram. In the case of an IID disorder, $h_{c}^{\text {que }}(\beta)<h_{c}(0)$ for all $\beta>0$ (see [3]), showing that disorder helps for localization: indeed, a typical renewal trajectory may target positive $\omega$ 's. Also, $h_{c}^{\text {que }}(\beta) \geq h_{c}^{\mathrm{a}}(\beta)$, see Section 1.4 (note that under some condition $h_{c}(0)-h_{c}^{\mathrm{a}}(\beta) \sim c s t . \beta^{2}$, see $(17)$ ). One can also show that there exists some $c>0$ such that $h_{c}^{\text {que }}(\beta) \leq \bar{h}(\beta)=h_{c}(0)-c \beta^{2}$, see $[25, \S 5.2]$ (the method is presented in the IID case, but is very general). When $\beta \rightarrow \infty$, one is able to get the asymptotic behavior $h_{c}^{\text {que }}(\beta) \stackrel{\beta \uparrow \infty}{\sim} \frac{1}{1+\alpha} h_{c}^{\mathrm{a}}(\beta)$ whenever the annealed model is defined for all $\beta>0$, as in [41, Eq. (3.8)] or [7, Prop. 5.2.4]. 
Another important feature of the model is the order of the phase transition: one desires to know how fast the free energy vanishes when $h$ approaches $h_{c}(\beta)$. Formally, one defines

$$
\nu^{\text {que }}:=\lim _{h \downarrow h_{c}^{\text {que }}(\beta)} \frac{\log \mathrm{F}(\beta, h)}{\log \left(h-h_{c}^{\text {que }}(\beta)\right)}
$$

if the limit exists, so that one has that $\mathrm{F}(\beta, h) \asymp\left(h-h_{c}(\beta)\right)^{\nu^{\text {que }}+o(1)}$ as $h \downarrow h_{c}(\beta)(a \asymp b$ means that the ratio $a / b$ is uniformly bounded away from 0 and $+\infty)$. The exponent $\nu^{\text {que }}$ reflects the growth of the contact fraction, see (4): if $\nu^{\text {que }} \leq 1$, then there is a jump discontinuity in the contact fraction, and if $\nu^{\text {que }}>1$, the contact fraction grows roughly as $\left(h-h_{c}(\beta)\right)^{\nu^{\text {que }}-1}$.

Aside from the free energy, another central physical quantity of disordered systems is the correlation length $\ell(\beta, h)=1 / r(\beta, h)$, where $r(\beta, h)$ is the exponential decay rate of "correlations" in the system. In the context of pinning model, it is natural to define, for $h>h_{c}(\beta)$,

$$
r(\beta, h):=\lim _{k \rightarrow \infty}-\frac{1}{k} \log \left(\mathbf{E}_{\infty, h}^{\beta, \omega}\left[\mathbf{1}_{\{i \in \tau\}} \mathbf{1}_{\{i+k \in \tau\}}\right]-\mathbf{E}_{\infty, h}^{\beta, \omega}\left[\mathbf{1}_{\{i \in \tau\}}\right] \mathbf{E}_{\infty, h}^{\beta \omega}\left[\mathbf{1}_{\{i+k \in \tau\}}\right]\right) \quad \mathbb{P}-\text { a.s. }
$$

where we denoted $\mathbf{E}_{\infty, h}^{\beta, \omega}[A]:=\lim _{N \rightarrow \infty} \mathbf{E}_{N, h}^{\beta, \omega}[A]$, which is known to exist $\mathbb{P}$-a.s. for any bounded local observable $A$ when $h>h_{c}^{\text {que }}(\beta)$, see $[30]$.

It is known that $r(0, h)=\mathrm{F}(0, h)$, see [26]. In the disordered case $(\beta>0)$, F. Toninelli [39, Thm. 3.5] also showed that $r(\beta, h)=\mathrm{F}(\beta, h)$, in the special case where the underlying renewal is the return times of a simple symmetric random walk $S$ in dimension 1 . The argument, however, works for any nearest-neighbor random walk in dimension 1, such as Bessel-type random walks [2]: we can therefore interpret the inverse of the free energy as the correlation length, for a wide class of renewals (with asymptotically any $\varphi(\cdot)$ and $\alpha \geq 0$ in Assumption 1.1).

The general goal is now to describe this pinning/depinning phase transition: can one compute explicitly or have sharp estimates on the critical point $h_{c}^{\text {que }}(\beta)$ ? what is the behavior of the free energy $\mathrm{F}(\beta, h)$ close to criticality (when $h \downarrow h_{c}^{\text {que }}(\beta)$ ), and in particular can one find $\nu^{\text {que? }}$ can one describe precisely path properties in the localized or delocalized phase? To that end, one compares the disordered system with its homogeneous and annealed counterparts, that we now define.

\subsection{The homogeneous model}

The homogeneous model is the non-disordered model, i.e. when $\beta=0$, and its partition function reads $Z_{N, h}^{\beta=0, \omega}=: Z_{N, h}^{\text {hom }}=\mathbf{E}\left[e^{h \sum_{n=1}^{N} \mathbf{1}_{\{n \in \tau\}}} \mathbf{1}_{\{N \in \tau\}}\right]$. This model is exactly solvable: one has an explicit expression for the free energy, and can derive from it the value of the critical point and the critical behavior of the free energy. The path properties can be described in details as well, cf. [25, Ch. 2] or [18, Ch. 7]: under $\mathbf{P}_{N, h}^{\beta=0}, \tau$ is a renewal with inter-arrival distribution $\widetilde{\mathrm{K}}_{h}(n)=e^{h} \mathrm{~K}(n) e^{-\mathrm{F}(0, h) n}$, conditioned to have $N \in \tau$.

Theorem 1.3 (cf. [25], Ch. 2). The homogeneous free energy $\mathrm{F}(0, h)$ is the solution in $x$ of the equation

$$
\sum_{n \in \mathbb{N}} \mathrm{K}(n) e^{-x n}=e^{-h}
$$

if a solution exists, and $\mathrm{F}(0, h)=0$ otherwise.

From (6), one deduces that the critical point is $h_{c}(0)=-\log \left(\mathbf{P}\left(\tau_{1}<+\infty\right)\right) \geq 0$. In particular, one has that $h_{c}(0)=0$ if and only if $\tau$ is recurrent. One also has the critical behavior of the free energy: for every $\alpha>0$ and $\varphi(\cdot)$, there exists an explicit slowly varying function $\widehat{\varphi}(\cdot)$ such that

$$
\mathrm{F}\left(0, h_{c}(0)+u\right) \stackrel{u \downarrow 0}{\sim} \widehat{\varphi}(1 / u) u^{\nu^{\mathrm{hom}}}
$$


where $\nu^{\text {hom }}=\max (1,1 / \alpha)$ is the order of the homogeneous phase transition (see (5)). When $\alpha=0, \mathrm{~F}\left(0, h_{c}(0)+\right.$ u) vanishes faster than any power of $u$, and the critical behavior is also explicit.

Assumption 1.4. In the following, we assume without loss of generality that the underlying renewal is recurrent, so that $h_{c}(0)=0$. Indeed, considering the inter-arrival distribution $\overline{\mathrm{K}}(n):=\mathrm{K}(n) / \mathbf{P}\left(\tau_{1}<+\infty\right)$ which is that of a recurrent renewal, one has that $\frac{\mathrm{d} \overline{\mathbf{P}}}{\mathrm{d} \mathbf{P}}(\tau \cap[0, N])=\left(\mathbf{P}\left(\tau_{1}<+\infty\right)\right)^{-|\tau \cap(0, N]|}$, so that one can re-write $(2)$ as

$$
Z_{N, h}^{\beta, \omega}=\overline{\mathbf{E}}\left[\exp \left(\sum_{n=1}^{N}\left(\beta \omega_{n}+h+\log \mathbf{P}\left(\tau_{1}<+\infty\right)\right) \mathbf{1}_{\{n \in \tau\}}\right) \mathbf{1}_{\{N \in \tau\}}\right] .
$$

Hence, replacing $\tau$ by a recurrent renewal with inter-arrival distribution $\bar{K}(\cdot)$ in $(2)$ results only in a shift in the parameter $h$ by $\log \mathbf{P}\left(\tau_{1}<+\infty\right)$.

\subsection{The annealed model}

The partition function, when averaged with respect to disorder (i.e. $\mathbb{E} Z_{N, h}^{\beta, \omega}$ ), is referred to as the annealed model. In the pinning model, one has

$$
\mathbb{E} Z_{N, h}^{\beta, \omega}=\mathbf{E}\left[e^{h \sum_{n=1}^{N} \mathbf{1}_{\{n \in \tau\}}} \mathbb{E}\left[e^{\beta \sum_{n=1}^{N} \omega_{n} \mathbf{1}_{\{n \in \tau\}}}\right] \mathbf{1}_{\{N \in \tau\}}\right]
$$

and the annealed measure $\mathbf{P}_{N, h}^{\beta, \text { a }}$ is defined accordingly. One also has the annealed free energy,

$$
\mathrm{F}^{\mathrm{a}}(\beta, h):=\liminf _{N \rightarrow \infty} \frac{1}{N} \log \mathbb{E} Z_{N, h}^{\beta, \omega}
$$

and its annealed critical point, $h_{c}^{\mathrm{a}}(\beta):=\inf \left\{h ; \mathrm{F}^{\mathrm{a}}(\beta, h)>0\right\}$. Then, Jensen's inequality gives that $\mathrm{F}(\beta, h)=$ $\lim _{N \rightarrow \infty} \frac{1}{N} \mathbb{E} \log Z_{N, h}^{\beta, \omega} \leq \mathrm{F}^{\mathrm{a}}(\beta, h)$, so that $h_{c}^{\text {que }}(\beta) \geq h_{c}^{\mathrm{a}}(\beta)$.

For the comparison between the quenched and annealed system to be of interest, one needs the annealed model to be non-degenerated. If the following assumption is not satisfied, then one has that $\mathrm{F}^{\mathrm{a}}(\beta, h)=+\infty$, and the inequality $\mathrm{F}(\beta, h) \leq \mathrm{F}^{\mathrm{a}}(\beta, h)$ is trivial.

Assumption 1.5. There exists some $\beta_{0}>0$ such that,

$$
\liminf _{N \rightarrow \infty} \frac{1}{N} \log \mathbb{E}\left[\exp \left(\beta \sum_{n=1}^{N} \omega_{n}\right)\right]<+\infty \quad \text { for all } \beta \leq \beta_{0} .
$$

In the IID case, it corresponds to asking that $\omega_{1}$ has a finite exponential moment for $\beta$ small enough,

$$
M(\beta):=\mathbb{E}\left[e^{\beta \omega_{1}}\right]<+\infty \quad \text { for all } \beta \leq \beta_{0} .
$$

We stress that if the $\omega_{n}$ 's are IID, then the annealed partition function is simply the partition function of an homogeneous model, with $h$ replaced by $h+\log M(\beta)$, so that $h_{c}^{\mathrm{a}}(\beta)=-\log M(\beta)$. The annealed system is then exactly solvable, and well understood, making it a cornerstone in the study of the influence of an IID disorder. When the $\omega_{n}$ 's are not IID anymore, the annealed system is not explicit, except in the case where $\left(\omega_{n}\right)_{n \in \mathbb{Z}}$ is a Gaussian stationary sequence with correlation function $\left(\rho_{k}\right)_{k \in \mathbb{Z}}, \rho_{k}:=\mathbb{E}\left[\omega_{0} \omega_{k}\right], \rho_{k}=\rho_{-k}$, for which the annealed partition function is

$$
\mathbb{E} Z_{N, h}^{\beta, \omega}=\mathbf{E}\left[\exp \left(h \sum_{n=1}^{N} \mathbf{1}_{\{n \in \tau\}}+\frac{\beta^{2}}{2} \sum_{1 \leq i, j \leq N} \rho_{j-i} \mathbf{1}_{\{i \in \tau\}} \mathbf{1}_{\{j \in \tau\}}\right) \mathbf{1}_{\{N \in \tau\}}\right] .
$$

One realizes that this is not the partition function of a standard homogeneous model, and its study is already challenging. 


\subsection{Disorder relevance/irrelevance?}

The central question to be answered is that of the influence of an arbitrarily small disorder on the phase transition. We compare the disordered and homogeneous (or annealed) phase transitions, and we determine whether their properties differ, in particular in terms of critical exponents (this is the original question of disorder relevance), but also in terms of critical points.

- Disorder is said to be relevant if for all $\beta>0$ the critical points and critical exponents differ: $h_{c}(\beta)>$ $h_{c}^{\mathrm{a}}(\beta) ; \nu^{\text {que }} \neq \nu^{\text {hom }}$.

- Disorder is said to be irrelevant if there exists some $\beta_{1}>0$ such that the critical points and critical exponents are equal for $\beta \leq \beta_{1}: h_{c}(\beta)=h_{c}^{\mathrm{a}}(\beta) ; \nu^{\text {que }}=\nu^{\text {hom }}$ for all $\beta \leq \beta_{1}$.

\section{ON THE INFLUENCE OF DISORDER IN THE IID CASE}

In the context of the disordered pinning model presented in Section 1 , we have that $\nu^{\text {hom }}=\max (1,1 / \alpha)$ : Harris' criterion therefore predicts that disorder is irrelevant if $\alpha<1 / 2$, and relevant if $\alpha>1 / 2$. The case $\alpha=1 / 2$, corresponding to the case of the simple symmetric random walk when $\varphi(\cdot)$ is a constant (see discussion after Assumption 1.1), is marginal.

\subsection{Results in the IID case}

In this section, we consider the case where $\left(\omega_{n}\right)_{n \in \mathbb{N}}$ is an IID sequence, which satisfies Assumption 1.5: the exponential moment is finite $M(\beta)<+\infty$ for $\beta$ smaller than some $\beta_{0}>0$, so that the annealed model is well defined. This case has been intensively studied in the past decade, and the Harris criterion is now settled, the marginal case $\alpha=1 / 2$ being also solved.

Theorem 2.1 (Irrelevant disorder). If $\alpha<1 / 2$, or if $\alpha=1 / 2$ and $\sum_{n \in \mathbb{N}} \frac{1}{n \varphi(n)^{2}}<+\infty$, then disorder is irrelevant: there exists some $\beta_{1}>0$ such that

- for all $\beta \leq \beta_{1}, h_{c}^{\text {que }}(\beta)=h_{c}^{\mathrm{a}}(\beta)$;

- for any $\beta \leq \beta_{1}$ and any $\eta>0$, there exists some $c_{\eta, \beta}$ such that, for all $u \leq c_{\eta, \beta}$,

$$
(1+\eta) \geq \frac{\mathrm{F}\left(\beta, h_{c}^{\mathrm{a}}(\beta)+u\right)}{\mathrm{F}\left(0, h_{c}(0)+u\right)} \geq(1-\eta) .
$$

Moreover, $\beta_{1}<+\infty$ if $\alpha>0$ (and disorder is unbounded, see [40]), and $\beta_{1}=+\infty$ if $\alpha=0$.

There exist several proofs of Theorem 2.1, with very different techniques: a second moment method in [1,39], martingale techniques in [34], and a large deviation/variational formula approach in [17]. The case $\alpha=0$ is studied in depth in [5]: the annealed and quenched critical points are equal, whatever $\beta$ is.

Theorem 2.2 (Relevant disorder). If $\alpha>1 / 2$, or if $\alpha=1 / 2$ and $\sum_{n \in \mathbb{N}} \frac{1}{n \varphi(n)^{2}}=+\infty$, then disorder is relevant: for every $\beta>0$, one has that

- the critical points differ, $h_{c}^{\text {que }}(\beta)>h_{c}^{\mathrm{a}}(\beta)$. Moreover, one has that there exists a slowly varying function $\psi(\cdot)$ (depending explicitly on $\alpha, \varphi(\cdot)$ ), some $\gamma:=\min \left\{\frac{2 \alpha}{2 \alpha-1}, 2\right\}$ and a constant $m_{\alpha}>0$ such that for $\alpha \in$ $(1 / 2,1) \cup(1,+\infty)$

$$
\lim _{\beta \downarrow 0} \frac{h_{c}^{\text {que }}(\beta)-h_{c}^{\mathrm{a}}(\beta)}{\psi(1 / \beta) \beta^{\gamma}}=m_{\alpha} .
$$

If $\alpha>1$, one has $\psi=1, \gamma=2$, and $m_{\alpha}=\frac{!}{2 \mathbf{E}\left[\tau_{1}\right]} \frac{\alpha}{1+\alpha}$.

In the case $\alpha=1 / 2$, a sharp asymptotic for the critical point shift is also given in [12]. For example, if $\lim _{n \rightarrow \infty} \varphi(n)=c_{\varphi}$, then one has that $\lim _{\beta \downarrow 0} \beta^{2} \log \left(h_{c}^{\text {que }}(\beta)-h_{c}^{\mathrm{a}}(\beta)\right)=-2 \pi^{2}\left(c_{\varphi}\right)^{2}$.

- the phase transition is of order at least $2, \nu^{\text {que }} \geq 2$. One refer to this phenomenon as the smoothing of the phase transition: close to criticality, the disordered free energy is smoother than the homogeneous one, since 
$\nu^{\text {que }} \geq 2>\nu^{\text {hom }}$ if $\alpha>1 / 2$. More precisely, for all $\beta>0$ and $v>0$, there exists a constant $C_{\beta, v}$, with $\lim _{\beta \downarrow 0, v \downarrow 0} C_{\beta, v}=1$ such that, for all $u \leq v$,

$$
\mathrm{F}\left(\beta, h_{c}^{\text {que }}(\beta)+u\right) \leq C_{\beta, v} \frac{1+\alpha}{2 \beta^{2}} u^{2} .
$$

Theorem 2.2 collects several results proven in a series of papers $[4,10,16,17,19,28,29,31]$ in several manners, the full necessary and sufficient condition for disorder relevance (together with the sharp critical point shift when $\alpha=1 / 2$ ) being given only recently in [12]. In [12,19,28,29], the authors estimate the fractional moment of the partition function up to the correlation length by a change of measure argument, and then use a coarse-graining procedure to glue these estimates together. A coarse-graining argument is also in order in [4], whereas in [17], a large deviation approach is used, enabling the authors to give a variational formula for the quenched critical point (and as a matter of fact, also for the annealed one). In $[15,31]$, the authors prove the smoothing of the free energy (12) via a rare-stretch strategy, that we outline in Section 2.2: it stresses the influence of rare regions in disorder relevance. For a more complete overview of the results and techniques employed, we refer the reader to $[27]$.

Let us now make a few miscellaneous comments.

1. Disorder relevance is monotone in $\beta$. It has been proven in [17] and in $[29, \S 6]$ that the critical point shift $\beta \mapsto h_{c}^{\text {que }}(\beta)-h_{c}^{\mathrm{a}}(\beta)$ is non-decreasing, so that disorder relevance is monotone in $\beta$. In particular, there exists a critical value $\beta_{c} \geq 0$ such that $h_{c}^{\text {que }}(\beta)=h_{c}^{\mathrm{a}}(\beta)$ if $\beta \leq \beta_{c}$, and $h_{c}^{\text {que }}(\beta)>h_{c}^{\mathrm{a}}(\beta)$ if $\beta>\beta_{c}$. Having that $\beta_{c}=0$ is therefore interpreted as the fact that disorder is relevant.

2. The order of the disordered phase transition is still unknown. When disorder is relevant, one obtains that the order of the phase transition is larger than the homogeneous one, $\nu^{\text {que }} \geq 2 \geq \nu^{\text {hom }}$ (with strict inequality if $\alpha>1 / 2$ ). But more precise results are lacking, and the mystery concerning the order of the phase transition remains, even at the heuristic level. However, recently, Derrida and Retaux [21] studied a toy hierarchical pinning model, for which the quenched critical point is explicit, and they show that the disordered phase transition has an essential singularity of the type $e^{-c s t .} / \sqrt{h-h_{c}(\beta)}$, so that $\nu^{\text {que }}=+\infty$. Even though their techniques rely strongly on the hierarchical structure, making the model more tractable (i.e. with a tractable renormalization group), it gives a first glimpse that the disordered phase transition should be of infinite order as soon as disorder is relevant.

3. Historical controversy about the marginal case $\alpha=1 / 2$. The marginal case $\alpha=1 / 2$, when the slowly varying function $\varphi(\cdot)$ is asymptotically equivalent to a constant (which is the case of a simple random walk on $\mathbb{Z}$ ), has long been subject to debates among physicists, with two different approaches and conjectures. In [24], the authors suggested that disorder were irrelevant, but then in [20], it was predicted that disorder were relevant, with a critical point shift of order $e^{-\mathrm{c} / \beta^{2}}$. Both predictions found supporters among the physics literature. In [28] the authors proved [20] right, and it is very recently in [12] that the conjecture that, for $\alpha=1 / 2$, disorder is relevant if and only if $\sum_{n \in \mathbb{N}} \frac{1}{n \varphi(n)^{2}}=+\infty$ was proven. In [12], the authors also obtain the sharp critical point shift $e^{-\mathrm{c} / \beta^{2}}$, the constant $\mathrm{c}$ being explicit: $\mathrm{c}=\pi / 2$ in the case of the simple random walk on $\mathbb{Z}$, which was the value conjectured in $[20]$.

Remark 2.3. The condition $\sum_{n \in \mathbb{N}} \frac{1}{n \varphi(n)^{2}}<+\infty$ appears naturally, since under Assumption 1.1, it is equivalent to the transience of the intersection $\tau \cap \tau^{\prime}$ of two independent copies of the renewal. Indeed, the average number of points in $\tau \cap \tau^{\prime}$ is $\sum_{n \geq 1} \mathbf{P}\left(n \in \tau \cap \tau^{\prime}\right)=\sum_{n \geq 1} \mathbf{P}(n \in \tau)^{2}$, and [22, Thm. B] gives (if $\left.0<\alpha<1\right)$ that $\mathbf{P}(n \in \tau) \stackrel{n \rightarrow \infty}{\sim} c_{\alpha} \varphi(n)^{-1} n^{-(1-\alpha)}$ for some constant $c_{\alpha}$. 
The intersection $\tau \cap \tau^{\prime}$ appears for instance when one computes the second moment of the partition function. For $h=h_{c}^{\mathrm{a}}(\beta)$, one gets

$$
\begin{array}{r}
\mathbb{E}\left[\left(Z_{N, h=h_{c}^{\mathrm{a}}(\beta)}^{\beta, \omega}\right)^{2}\right]=\mathbb{E}\left[\mathbf{E}^{\otimes 2}\left[\exp \left(\sum_{n=1}^{N}\left(\beta \omega_{n}-\log M(\beta)\right)\left(\mathbf{1}_{\{n \in \tau\}}+\mathbf{1}_{\left\{n \in \tau^{\prime}\right\}}\right)\right) \mathbf{1}_{\{N \in \tau\}} \mathbf{1}_{\left\{N \in \tau^{\prime}\right\}}\right]\right] \\
=\mathbf{E}^{\otimes 2}\left[\exp \left(\sum_{n=1}^{N}(\log M(2 \beta)-2 \log M(\beta)) \mathbf{1}_{\left\{n \in \tau \cap \tau^{\prime}\right\}}\right) \mathbf{1}_{\left\{N \in \tau \cap \tau^{\prime}\right\}}\right]
\end{array}
$$

which is the partition function of an homogeneous system, with underlying renewal $\tau \cap \tau^{\prime}$. Therefore, as $N \rightarrow \infty$, (13) diverges for all $\beta>0$ if $\tau \cap \tau^{\prime}$ is recurrent, and remains bounded if $\tau \cap \tau^{\prime}$ is transient provided that $\beta$ is small enough, in which case there is a chance that the quenched and annealed system are close.

As far as path properties under $\mathbf{P}_{N, h}^{\beta, \omega}$ are concerned, we have already seen that one can extract the density of contacts from the free energy, and it is $\partial_{h} \mathrm{~F}(\beta, h)>0$ in the localized phase. In the delocalized phase, the answer is however not trivial. For the homogeneous case, one knows that the number of contact remains bounded if $h<h_{c}(0)$ [27, Ch. 7], whereas in the disordered case the situation is more subtle, since there might be some stretches of unusual disorder driving the polymer to have a growing number of contacts, even if $h<h_{c}^{\text {que }}(\beta)$. One a priori only knows that the number of contacts is $o(N)$ in the delocalized regime, but [35] gives that the number of contact under $\mathbf{P}_{N, h}^{\beta, \omega}$ is $O(\log N) \mathbb{P}$-a.s. In [6], the authors show that the last contact occurs at distance $O(1)$ of the origin in probability (cf. Thm. 1.1), but they exhibit some parameters ( $\beta$ large enough, some $\left.h_{c}^{\mathrm{a}}(\beta)<h<h_{c}^{\text {que }}(\beta)\right)$ for which the number of contacts is exactly of order $\log N \mathbb{P}$-a.s.

Remark 2.4. One can wonder what happens if Assumption 1.5 is not satisfied, so that $\mathrm{F}^{\mathrm{a}}(\beta, h)=+\infty$ for all $\beta>0, h \in \mathbb{R}$. In the IID setting, it corresponds to the case when $\omega_{1}$ does not admit any exponential moment, $\mathbb{E}\left[e^{\beta \omega_{1}}\right]=+\infty$ for all $\beta>0$. In that case, one proves that the behavior of the quenched system is also degenerated: one can show that there is no phase transition anymore, the quenched critical point being $h_{c}^{\text {que }}(\beta)=-\infty$. One is also able to obtain the behavior of the free energy as $h$ goes to $-\infty, \mathrm{F}(\beta, h) \stackrel{h \rightarrow \infty}{\sim}$ $\beta \int_{x \geq|h| / \beta} \mathbb{P}\left(\omega_{1} \geq x\right) \mathrm{d} x$.

\subsection{The rare-stretch strategy and smoothing of the free energy}

We now stress the importance of rare regions in disorder relevance and in particular in the smoothing of the free energy curve (12), by sketching the rare stretch strategy. One divides the system in regions of size $m$, and denote $B_{i}:=\{(i-1) m+1, \ldots, i m\}$ the $i^{\text {th }}$ block. One also defines the property $\mathcal{A}_{m}=\mathcal{A}\left(\omega_{1}, \ldots, \omega_{m}\right)$ of being favorable (definition which has to be made specific at some point). Then, the rare-stretch strategy consists in imposing the renewal $\tau$ to visit only the blocks $B_{i}$ for $i \in \mathcal{I}$, where $\mathcal{I}=\left\{i ;\left(\omega_{k}\right)_{k \in B_{i}} \in \mathcal{A}\right\}$ are the indices of the favorable blocks, see Figure 3: this gives a non-trivial lower bound on the free energy.

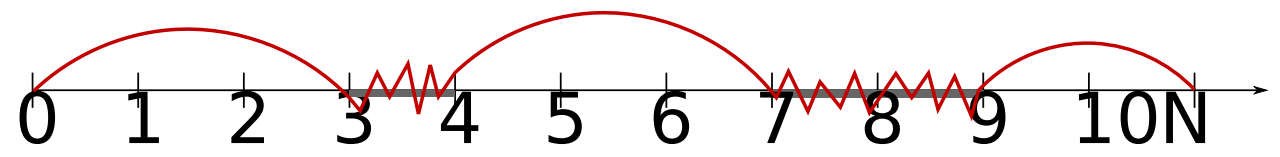

FIGURE 3. Overview of the rare-stretch strategy. Here, $N=11 \mathrm{~m}$, and the underlined regions are the ones identified as favorable: $\mathcal{I}=\{3,7,8\}$. Then, we impose the renewal trajectory to visit only these regions.

Then, loosely speaking, the energetic gain (at the exponential level) on a favorable region is $\mathbb{E}\left[\log Z_{m, h}^{\beta, \omega} \mid \mathcal{A}_{m}\right]$. On the other hand, since the first favorable region is at distance $\approx m \mathbb{P}\left(\mathcal{A}_{m}\right)^{-1}$, the entropic cost of aiming at a favorable block is $\log \mathrm{K}\left(m \mathbb{P}\left(\mathcal{A}_{m}\right)^{-1}\right) \approx-(1+\alpha) \log \left(\mathbb{P}\left(\mathcal{A}_{m}\right)^{-1}\right)$. Therefore, in a system constituted of $n$ 
blocks, one roughly has that $\log Z_{n m} \geq|\mathcal{I} \cap[0, n]| \times\left(\mathbb{E}\left[\log Z_{m, h}^{\beta, \omega} \mid \mathcal{A}_{m}\right]-(1+\alpha) \log \left(\mathbb{P}\left(\mathcal{A}_{m}\right)^{-1}\right)\right)$, and when taking $\lim _{n \rightarrow \infty} \frac{1}{m n} \log Z_{n m}$ one obtains

$$
\mathrm{F}(\beta, h) \geq \mathbb{P}\left(\mathcal{A}_{m}\right)\left(\frac{1}{m} \mathbb{E}\left[\log Z_{m, h}^{\beta, \omega} \mid \mathcal{A}_{m}\right]-(1+\alpha) \frac{1}{m} \log \left(\mathbb{P}\left(\mathcal{A}_{m}\right)^{-1}\right)\right)
$$

In the end, the key is to define properly $\mathcal{A}_{m}$, in order to optimize the lower bound (14).

For example, to obtain the smoothing of the free energy, the idea is to set, for $u \geq 0$, the property $\mathcal{A}_{m}=$ $\left\{\left(\omega_{1}, \ldots, \omega_{m}\right)\right.$ has empirical mean $\left.u / \beta\right\}$. Then, one naturally has that $\lim _{m \rightarrow \infty} \frac{1}{m} \mathbb{E}\left[\log Z_{m, h}^{\beta, \omega} \mid \mathcal{A}_{m}\right]=\mathrm{F}(\beta, h+u)$. Also, $\mathbb{P}\left(\mathcal{A}_{m}\right)$, the probability that the $\left(\omega_{i}\right)_{1 \leq i \leq m}$ are shifted by $u / \beta$, is approximately $e^{-C_{\beta, u} \frac{u^{2}}{2 \beta^{2}} m}$, cf. [15]. Finally, setting $h=h_{c}^{\text {que }}(\beta)$ so that $\mathrm{F}\left(\beta, h_{c}^{\text {que }}(\beta)\right)=0$, one obtains in (14), letting $m$ go to infinity,

$$
0 \geq \mathrm{F}\left(\beta, h_{c}^{\text {que }}(\beta)+u\right)-(1+\alpha) C_{\beta, u} \frac{u^{2}}{2 \beta^{2}}
$$

\section{CASE OF A CORRElated Disorder}

Once that one has studied the case of an IID environment, it is natural to turn to a correlated one, with two-point correlations in $\omega$ decaying with the distance as a power law $|i-j|^{-\xi}$. In the context of the pinning model, Weinrib and Halperin prediction [43] tells that disorder should be irrelevant if $\nu^{\text {hom }}>2 \mathrm{~min}(1, \xi)$, that is if $\alpha<\min (1, \xi) / 2$.

\subsection{Gaussian environment with long-range correlations}

\subsubsection{The annealed model}

Since the annealed model is in general not explicit, the first step is to work with a stationary Gaussian environment, with correlation function $\left(\rho_{k}\right)_{k \in \mathbb{Z}}$, for which the annealed partition function is, as mentioned in Section 1.4,

$$
\mathbb{E} Z_{N, h}^{\beta, \omega}=\mathbf{E}\left[\exp \left(h \sum_{n=1}^{N} \mathbf{1}_{\{n \in \tau\}}+\frac{\beta^{2}}{2} \sum_{1 \leq i, j \leq N} \rho_{j-i} \mathbf{1}_{\{i \in \tau\}} \mathbf{1}_{\{j \in \tau\}}\right) \mathbf{1}_{\{N \in \tau\}}\right]
$$

Here, Assumption 1.5 reads $\sum_{n \in \mathbb{Z}}\left|\rho_{n}\right|<+\infty$ (recall condition (8)), and it is shown that the annealed free energy $\mathrm{F}^{\mathrm{a}}(\beta, h)$ exists and if finite under that condition. Remark that, if $\rho_{n} \stackrel{n \rightarrow \infty}{\sim} n^{-\xi}$ for some $\xi>0$, the condition $\sum_{n \in \mathbb{Z}}\left|\rho_{n}\right|<+\infty$ implies in particular that $\xi>1$.

This annealed model is highly non-trivial, and it has been studied in $[8,36]$ via two different approaches. We collect here the main results.

Theorem 3.1. Even though the critical point is not explicit, whenever $\sum_{n \in \mathbb{Z}}\left|\rho_{n}\right|<+\infty$ (or $\xi>1$ ), one has

$$
h_{c}^{\mathrm{a}}(\beta) \stackrel{\beta \downarrow 0}{\sim}-\frac{1}{2} \beta^{2}\left(1+2 \sum_{n \in \mathbb{N}} \rho_{n} \mathbf{P}(n \in \tau)\right) .
$$

If moreover $\sum_{n \in \mathbb{N}} n\left|\rho_{n}\right|<+\infty$ (or $\xi>2$ ), then the annealed critical behavior is similar to that of the homogeneous system: for any $\beta>0$, there exists some constant $c_{\beta} \in(0,1)$ such that, for all $u \in[0,1]$, one has

$$
c_{\beta} \mathrm{F}(0, u) \leq \mathrm{F}^{\mathrm{a}}\left(\beta, h_{c}^{\mathrm{a}}(\beta)+u\right) \leq \frac{1}{c_{\beta}} \mathrm{F}(0, u)
$$




\subsubsection{Disorder relevance/irrelevance}

If $\sum_{n \in \mathbb{N}}\left|\rho_{n}\right|<+\infty$ (or $\xi>1$ ), then according to the Weinrib-Halperin predictions, one expects the disorder relevance/irrelevance picture to be similar to the IID case. Several partial results are now available in this direction.

The smoothing of the free energy analogue of (12) is also obtained, showing disorder relevance in terms of critical exponents when $\alpha>1 / 2$ (since one obtains $\nu^{\text {que }} \geq 2>\nu^{\text {hom }}$ ), as in the IID case.

Proposition 3.2 (Theorem 5.6, [8]). If $\sum_{n \in \mathbb{N}}\left|\rho_{n}\right|<+\infty$ (or $\xi>1$ ), one has that for any $\beta>0$ and $u \geq 0$,

$$
\mathrm{F}\left(\beta, h_{c}^{\text {que }}(\beta)+u\right) \leq \frac{1}{\sum_{n \in \mathbb{Z}} \rho_{n}} \times \frac{1+\alpha}{2 \beta^{2}} u^{2}
$$

The critical point shift when $\alpha>1 / 2$ or equality when $\alpha<1 / 2$ has not been proven in full generality in the correlated context. The only result is when $\mathbf{E}\left[\tau_{1}\right]<+\infty$, where, remarkably, one is able to get the asymptotic behavior of the quenched critical point [13, Thm. 9], as in the IID case (Theorem 2.2):

$$
\lim _{\beta \downarrow 0} \frac{1}{\beta^{2}}\left(h_{c}^{\text {que }}(\beta)-h_{c}^{\mathrm{a}}(\beta)\right)=\sum_{n \in \mathbb{Z}} \rho_{n} \times \frac{1}{2 \mathbf{E}\left[\tau_{1}\right]} \frac{\alpha}{1+\alpha} .
$$

Remark 3.3. In [14], the authors study the hierarchical counterpart of this Gaussian correlated disordered pinning model. The hierarchical structure enables them to control the annealed measure close to criticality, without any knowledge on the annealed critical point: this trick works whenever $\xi>1$, with the restriction that $\xi<2 \min (1, \alpha)$. In particular, their results suggest that the annealed critical behavior should be different than the homogeneous one when $\xi<2 \min (1, \alpha)$. In [14], the authors show disorder irrelevance when $\alpha<1 / 2$, in terms of critical points and critical behavior, and are able to show the critical point shift if $\xi>1, \xi<2 \mathrm{~min}(1, \alpha)$. We collect the results in the hierarchical and non-hierarchical context in Figure 4.

\subsubsection{Non-summable correlations}

The most interesting part comes when one considers non-summable correlations, or in Weinrib Halperin words, if $\rho_{n} \stackrel{n \rightarrow \infty}{\sim} n^{-\xi}$ with $\xi<1$, when the Harris prediction should be modified, and disorder should be relevant when $\alpha>\xi / 2$ (instead of when $\alpha>1 / 2$ in the case $\xi>1$ ). We already know that Assumption 1.5 is not satisfied: the annealed model is degenerated, $\mathrm{F}^{\mathrm{a}}(\beta, h)=+\infty$ for all $h \in \mathbb{R}, \beta>0$. We show that the disordered model is actually also degenerated, in the sense that there is no phase transition: the system is always in the localized phase, $h_{c}^{\text {que }}(\beta)=-\infty$.

Theorem 3.4 (Thm. 6, [8]). If $\rho_{n} \stackrel{n \rightarrow \infty}{\sim} n^{-\xi}$ with $\xi<1$, then there exist constants $c_{1}>0$ such that, for all $\beta>0$ and $h \leq-1$,

$$
\mathrm{F}(\beta, h) \geq c_{1}^{-1} \exp \left(-c_{1} \beta^{2 /(1-\xi)}|h|^{-\xi /(1-\xi)}\right) .
$$

This is due to the fact that rare favorable regions appear much more frequently, because of stronger fluctuations of $\omega$. One can turn this idea into a proof, thanks to the rare-stretch strategy exposed in Section 2.2 .

Indeed, the inequality (14) is still valid. Then, if one defines $\mathcal{A}_{m}:=\left\{\omega_{i} \geq 2|h| / \beta\right.$; for $\left.1 \leq i \leq m\right\}$, so that $\beta \omega_{n}+h \geq|h|$ on favorable blocks, one obtains that $\mathbb{E}\left[\log Z_{m, h}^{\beta, \omega} \mid \mathcal{A}_{m}\right] \geq \log Z_{m,|h|}^{\text {hom }}$ which is approximately $m \mathrm{~F}(0,|h|) \geq c^{\prime \prime}|h| m$ if $h \leq-1$. It is then just a matter of estimating $\mathbb{P}\left(\mathcal{A}_{m}\right)$, the cost of shifting all $\omega$ 's so that they are all larger than $2|h| / \beta$ : Lemma 4.1 in [8] gives that $\mathbb{P}\left(\mathcal{A}_{m}\right) \geq e^{-c \frac{|h|^{2}}{\beta^{2}} m^{\xi}}$ if $\xi<1$. This is the crucial point, and stresses that rare regions are much more frequent than in the IID case. Plugged in (14), one gets that

$$
\mathrm{F}(\beta, h) \geq e^{-c \frac{|h|^{2}}{\beta^{2}} m^{\xi}}\left(c^{\prime \prime}|h|-c^{\prime} \frac{|h|^{2}}{\beta^{2}} m^{\xi-1}\right) .
$$

Now one just need to optimize the choice of $m$, taking $m^{\xi-1}=c s t . \beta^{2} /|h|$, to get (19). 


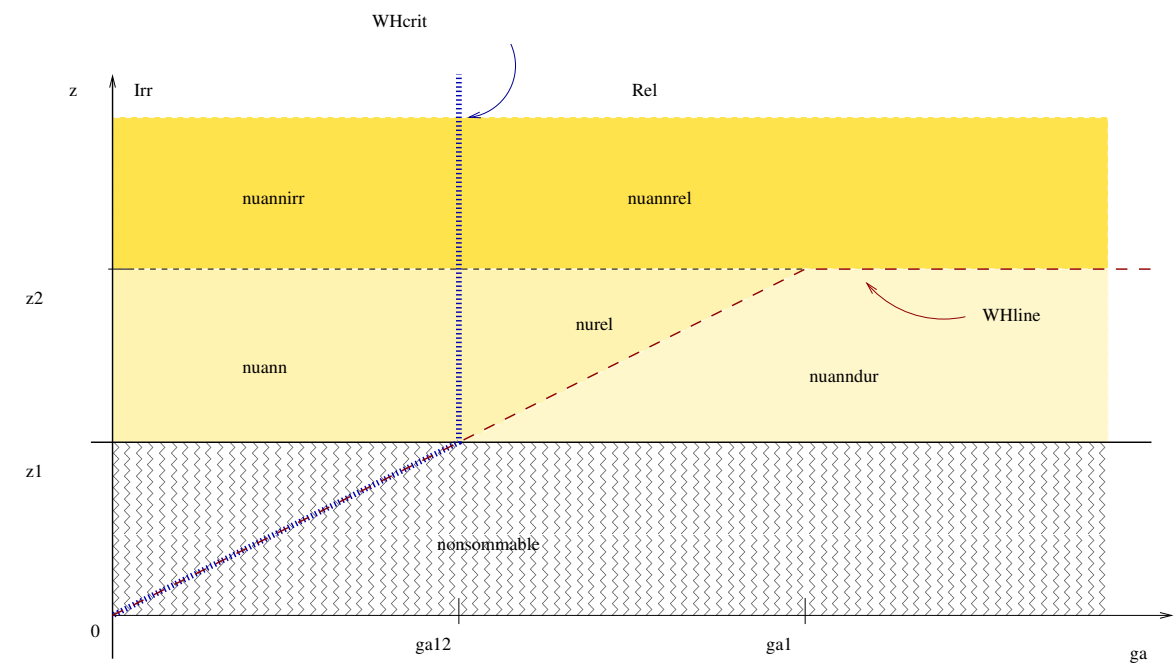

FIGURE 4. Overview of the results obtained in the standard and hierarchical pinning model with a Gaussian correlated environment with $\rho_{n} \stackrel{n \rightarrow \infty}{\sim} n^{-\xi}$. Weinrib and Halperin predict that disorder is irrelevant if $\alpha<$ $2 \min (1, \xi)$. First of all, the annealed and homogeneous critical behaviors are shown to be the same when $\xi>2$ (Theorem 3.1). In the hierarchical context, it has also been shown that that $\nu^{\mathrm{a}}=\nu^{\text {hom }}$ when $\xi>2 \min (1, \alpha)$, but there is some piece of evidence suggesting that $\nu^{\text {que }}>\nu^{\text {a }}$ when $1<\xi<2 \min (1, \alpha)$. When $\xi>1$, the smoothing of the free energy (19) shows that disorder is relevant for $\alpha>1 / 2$. In the hierarchical setting, disorder has also been proven to be irrelevant for $\alpha<1 / 2$ (equality of critical points and critical exponents for $\beta$ small enough). To summarize, Weinrib and Halperin prediction is validated when $\xi>1$ (fully in the hierarchical model, partially in the standard model). However, when $\xi<1$ the annealed model is degenerated, and the disordered system is always in the localized phase, see Section 3.1.3: disorder has a strong influence on the system whatever $\alpha>0$ is, and Weinrib Halperin prediction's is proven wrong.

\subsection{The infinite disorder regime}

As seen in previous sections, the effect of rare regions on critical properties is crucial, and reveals the relevant character of disorder. We saw in Theorem 3.4 and the discussion that followed, that having favorable regions of length $m$ only subexponentially rarely can drastically change the behavior of the system. Note that this phenomenon can occur even if Assumption 1.5 is satisfied, as studied in [9]. For example, take a sequence $\omega \in\{-1,1\}^{\mathbb{N}}$ (so that (8) is trivially satisfied), with $\mathbb{P}\left(\omega_{1}=+1, \ldots, \omega_{m}=+1\right.$ ) decaying subexponentially in $m$ : favorable regions of length $m$ with only +1 's occur only subexponentially rarely! This is known in the physics literature as the infinite disorder phenomenon, and can be defined properly in the context of the pinning model as follows.

Denote $\mathbf{M}:=\operatorname{ess} \sup \omega_{1}(\mathbf{M}=+\infty$ if $\omega$ is not bounded). Then, for any neighborhood $V$ of $\mathbf{M}$, we define the event $\mathcal{A}_{m}^{(V)}:=\left\{\omega_{i} \in V ; \forall 1 \leq i \leq m\right\}$, of having a " $V$-favorable" region of size $m$ : the frequency of their occurrence is $\mathbb{P}\left(\mathcal{A}_{m}^{(V)}\right)$.

Definition 3.5. One is said to have infinite disorder if

$$
\text { for any neighborhood } V \text { of } \mathbf{M} \text {, one has } \quad \liminf _{m \rightarrow \infty}-\frac{1}{m} \log \mathbb{P}\left(\mathcal{A}_{m}^{(V)}\right)=0 .
$$

It corresponds to having arbitrarily favorable regions occur only subexponentially rarely.

Rare regions effects has been studied in depth in the physical literature, in particular via a renormalization group approach, see [42] for a review. To use renormalization group philosophy, in the infinite disorder (or strong 
randomness) regime, atypical regions occur very frequently (as a matter of fact, are only subexponentially rare), and their impact on the system blows up when taking larger and larger length scales. This regime has been investigated for various systems, for which the strong randomness renormalization group may in general be carried out explicitly, and many exact results can be derived, see [33] for a brief review.

In the context of pinning model, we have seen a first occurrence of infinite disorder in Theorem 3.4. The above discussion suggests that

a) the central quantity characterizing infinite disorder is not the two-point correlation, but the frequency of occurrence of rare atypical regions;

b) whenever one lies in the infinite disorder regime, the phase transition is drastically modified, and one can hope to obtain exact results on the critical behavior of the system.

Point a) is made rigorous in [9] in the case of a binary disorder $\left(\omega_{i} \in\{-1,1\}\right)$, but its results holds with more generality.

Theorem 3.6 (Theorems 1.5-1.6 [9]). If one has infinite disorder, then $h_{c}^{\text {que }}(\beta)=-\beta \mathbf{M}$ for all $\beta>0$.

Moreover, one has explicit lower and upper bounds on $\mathrm{F}(\beta, h)$, which do not match in general, but give that disorder is relevant, whatever the value of $\alpha$ is: if $\mathbf{M}<+\infty$, then for all $\beta>0$

$$
\mathrm{F}\left(\beta, h_{c}^{\text {que }}(\beta)+u\right) \stackrel{u \downarrow 0}{=} o(\mathrm{~F}(0, u))
$$

Let us make some comments.

1. Weinrib and Halperin's predictions are not valid. First of all, one realizes that the modification of the Harris criterion does not occur when the correlations decay exponent is $\xi<1$, but with another weaker condition, (23). Secondly, when infinite disorder holds, Harris criterion is indeed modified, but not according to Weinrib and Halperin' prediction: one does not have a relevance $(\alpha>\xi / 2) /$ irrelevance $(\alpha<\xi / 2)$ picture anymore, and disorder is relevant for all $\alpha \geq 0$. It seems that there should be only two possible regimes: either there is no infinite disorder, in which case Harris criterion should be valid ; either one has infinite disorder, in which case Harris' relevance/irrelevance picture is altered, disorder being relevant whatever $\alpha$ is.

To illustrate this remark, we mention the work [11], in which the authors provide an example of a binary environment $\omega \in\{-1,0\}$, with long-range correlations $\mathbb{E}\left[\omega_{i} \omega_{i+k}\right] \sim k^{-\xi}$ with $\xi>0$, but such that $\mathbb{P}\left(\omega_{i}=\right.$ $0, \forall 1 \leq i \leq n) \stackrel{n \rightarrow \infty}{\sim} n^{-(2+\xi)}$. The environment is constituted of alternating blocks of 0's and -1 's, the lengths of the blocks being independent random variables, with heavy-tail distribution of exponent $2+\xi, \xi>0$. With this environment, one has infinite disorder $((23)$ holds) whatever the two point correlation decay exponent $\xi>0$ is. Therefore $h_{c}(\beta)=0$ for all $\beta>0$ (according to Theorem 3.6), and the sharp critical behavior of the free energy is found: $\mathrm{F}(\beta, u) \asymp|\log u|^{-\xi} \mathrm{F}(0, u)^{1+\xi}$. The order of the disordered phase transition is therefore computed exactly, $\nu^{\text {que }}=(1+\xi) \nu^{\text {hom }}>\nu^{\text {pur }}$, and remarkably, the correct logarithmic corrections are also found. Disorder is then relevant whatever $\xi>0$ and $\alpha$ are, disproving Weinrib and Halperin's prediction. According to the physics literature, one may hope to obtain the exact behavior of the free energy for more general models, whenever one lies in the infinite disorder regime, see Conjecture 7.3.3. in [7].

2. About the annealed model. Definition 3.5 of infinite disorder has also some consequences on the annealed system. Indeed, one has that $\mathbb{E} Z_{N, h}^{\beta, \omega} \geq \mathbb{P}\left(\mathcal{A}_{N}^{(V)}\right) Z_{N, \beta \text { inf } V+h}^{\text {hom }}$, so that when (23) holds, one gets that $\mathrm{F}^{\mathrm{a}}(\beta, h)=\mathrm{F}(0, \beta \mathbf{M}+h)$ : the annealed free energy is degenerated. This has to be related to Assumption 1.5 , since if (23) holds, one can obtain something similar to (8), namely

$$
\liminf _{N \rightarrow \infty} \frac{1}{N} \log \mathbb{E}\left[\exp \left(\beta \sum_{n=1}^{N} \omega_{n}\right)\right]=\beta \mathbf{M} \quad \text { for all } \beta>0 .
$$

It is then natural to ask whether the annealed and disordered models are non-degenerated whenever (23) does not hold. This is proven in [9], under some additional assumption that correlations are non-increasing (in some specific sense, see Assumption 2.2 in [9]), and we can conjecture that this is true in full generality. 


\section{ANOTHER PERSPECTIVE ON DISORDER RELEVANCE: SCALING LIMITS}

Another central question regarding disordered system is the one of the scaling limit: one might hope to be able to construct a continuum model which is the scaling limit of the system, in order to obtain for example universality results.

We consider in this section the case $\alpha \in(0,1)$, and give only the ideas of the results, for the sake of conciseness. Denote $\tau_{(N)}$ the renormalized set of contact points $\frac{1}{N} \tau \cap[0, N]$, which is a closed subset of $[0,1]$ (the set of closed subsets of $[0,1]$ is equipped with the Hausdorff topology). One has that $\tau_{(N)}$ converges in distribution towards the regenerative set with Lévy exponent $\alpha$, see [25, Thm. 2.7]. Analogously, one can consider the scaling limit of the pinning model: the idea is to take, as the size $N$ of the system goes to infinity, parameters $\beta_{N}$ and $h_{N}$ which scale properly with the system in order to obtain a non-trivial limit.

A first piece of work focuses on the scaling limit of the homogeneous pinning model, see [38]: there exists a critical window for the parameter $h$, for which the scaling limit is non-trivial. Let $b_{N}:=N^{\alpha} \varphi(N)^{-1}$, and set $h_{N}=\widehat{h} / b_{N}$ : one obtains that the set $\tau_{(N)}$, when distributed according to $\mathbf{P}_{N, h_{N}}$, converges in law to some specific limit $\mathcal{B}_{\alpha, \widehat{h}}$ which depends only on $\widehat{h}$ and $\alpha$, see [38, Thm. 3.1] for a precise statement.

The scaling limit of the disordered pinning model has been considered only recently, in [23], in the case of an IID disorder $\omega$. The authors show that, if $1 / 2<\alpha<1$, and setting $\beta=\widehat{\beta} N^{1 / 2} / b_{N}, h-h_{c}^{\mathrm{a}}(\beta)=\widehat{h} / b_{N}$, then for all $t \geq 0$, the partition function $Z_{t N, h}^{\beta, \omega}$ converges in distribution towards a non-trivial random variable $\mathbf{Z}_{t, \widehat{h}}^{\widehat{\beta}, \mathrm{W}}$ (which has an explicit dependence on the parameters), where $\mathrm{W}(\cdot)$ denotes the white noise on $\mathbb{R}$ : we refer to $[23$, Thm. 3.1] for a precise statement of the result. Their idea is to perform a so-called polynomial chaos expansion of the partition function $Z_{N, h}^{\beta, \omega}$, and prove the convergence of such an expansion. The remarkable fact about this result is that one obtains a disordered continuum limit if $\alpha>1 / 2$, and it is conjectured to be also the case when $\alpha=1 / 2$ and $\sum \frac{1}{n \varphi(n)^{2}}<+\infty$ : disorder survives when one takes the scaling limit, and it seems to survive exactly when disorder is relevant. This idea of having a disordered scaling limit is not directly related to disorder relevance as we discussed it in the previous sections (since the parameters go to 0), but it provides a

new approach to the question of the influence of disorder on physical systems, and gives rise to new interesting continuum models.

\section{REFERENCES}

[1] K. S. Alexander. The effect of disorder on polymer depinning transitions. Commun. Math. Phys., 279:117-146, 2008.

[2] K. S. Alexander. Excursions and local limit theorems for bessel-like random walks. Electr. Jour. Probab., 16:1-44, 2011.

[3] K. S. Alexander and V. Sidoravicius. Pinning of polymers and interfaces by random potentials. Ann. Appl. Probab., 16(2):636669, 2006.

[4] K. S. Alexander and N. Zygouras. Quenched and annealed critical points in polymer pinning models. Commun. Math. Phys., 291:659-689, 2009.

[5] K. S. Alexander and N. Zygouras. Equality of critical points for polymer depinning transitions with loop exponent one. Ann. Appl. Probab., 20(1):356-366, 2010.

[6] K. S. Alexander and N. Zygouras. Path properties of the disordered pinning model in the delocalized regime. Ann. Appl. Probab., 2:599-615, 2014.

[7] Q. Berger. Polymères en milieu aléatoire: influence d'un désordre corrélé sur le phénomène de localisation. PhD thesis, École Normale Supérieure de Lyon, 2012.

[8] Q. Berger. Comments on the influence of disorder for pinning model in gaussian correlated environment. ALEA, X(2):953-977, 2013.

[9] Q. Berger. Pinning model in random correlated environment: appearance of an infinite disorder regime. Jour. of Stat. Physics, 155(Issue 3):544-570, 2014.

[10] Q. Berger, F. Caravenna, J. Poisat, R. Sun, and N. Zygouras. The critical curves of the random pinning and copolymer models at weak coupling. Commun. Math. Phys., 326(2):507-530, 2014.

[11] Q. Berger and H. Lacoin. Sharp critical behavior for random pinning model with correlated environment. Stoch. Proc. Appl., 122(4):1397-1436, 2012. 
[12] Q. Berger and H. Lacoin. Pinning on a defect line: characterization of marginal disorder relevance and sharp asymptotics for the critical point shift. arXiv:1503.07315 [math-ph], 2015.

[13] Q. Berger and J. Poisat. On the critical curves of the copolymer and pinning model in correlated gaussian environment. arXiv:1404.5939 [math.PR], 2014.

[14] Q. Berger and F. L. Toninelli. Hierarchical pinning model in correlated random environment. Ann. Inst. H. Poincaré Probab. Stat., 48(3):781-816, 2013.

[15] F. Caravenna and F. den Hollander. A general smoothing inequality for disordered polymers. Electron. Commun. Probab., 18(76):1-15, 2013.

[16] F. Caravenna, F. Toninelli, and N. Torri. Universality for the pinning model in the weak coupling regime. arXiv:1505.04927 [math.PR], 2015.

[17] D. Cheliotis and F. den Hollander. Variational characterization of the critical curve for pinning of random polymers. Ann. Probab., 41:1767-1805, 2013.

[18] F. den Hollander. Random Polymers. Lecture notes in Mathematics - École d'Été de Probabilités de Saint-Flour XXXVII-2007. Springer, 2009.

[19] B. Derrida, G. Giacomin, H. Lacoin, and F. L. Toninelli. Fractional moment bounds and disorder relevance for pinning models. Commun. Math. Phys., 287:867-887, 2009.

[20] B. Derrida, V. Hakim, and J. Vannimenus. Effect of disorder on two-dimensional wetting. J. Stat. Phys., 66:1189-1213, 1992.

[21] B. Derrida and Martin Reteaux. The depinning transition in presence of disorder: a toy model. Jour. of Stat. Physics, 156(2), 2014.

[22] R. A. Doney. One-sided local large deviation and renewal theorems in the case of infinite mean. Probab. Theory Relat. Fields, 107:451-465, 1997.

[23] R. Sun F. Caravenna and N. Zygouras. Polynomial chaos and scaling limits of disordered systems. J. Eur. Math. Soc., to appear.

[24] G. Forgacs, J. M. Luck, T. M. Nieuwenhuizen, and H. Orland. Wetting of a disordered substrate: exact critical behavior in two dimensionsfor. Phys. Rev. Lett., 57:2184-2187, 1986.

[25] G. Giacomin. Random Polymer models. Imperial College Press, 2007.

[26] G. Giacomin. Renewal convergence rates and correlation decay for homogeneous pinning models. Elec. Jour. Probab., 13:513$529,2008$.

[27] G. Giacomin. Disorder and critical phenomena through basic probability models. Lecture notes in Mathematics - École d'Été de Probabilités de Saint-Flour XL-2010. Springer, lecture notes in mathematics edition, 2011.

[28] G. Giacomin, H. Lacoin, and F. L. Toninelli. Marginal relevance of disorder for pinning models. Commun. Pure Appl. Math., 63:233-265, 2010.

[29] G. Giacomin, H. Lacoin, and F. L. Toninelli. Disorder relevance at marginality and critical point shift. Ann. Inst. H. Poincaré, 47:148-175, 2011.

[30] G. Giacomin and F. L. Toninelli. The localized phase of disordered copolymers with adsorption. ALEA, 1:149-180, 2006.

[31] G. Giacomin and F. L. Toninelli. Smoothing effect of quenched disorder on polymer depinning transitions. Commun. Math. Phys., 266:1-16, 2006.

[32] A. B. Harris. Effect of random defects on the critical behaviour of ising models. J. Phys. C, 7:1671-1692, 1974.

[33] D. A. Huse. Renormalizing systems with strong quenched randomness. Physics Reports, 348:159-162, 2001.

[34] H. Lacoin. The martingale approach to disorder irrelevance for pinning models. Elec. Comm. Probab., 15:418-427, 2010.

[35] J.-C. Mourrat. On the delocalized phase of the random pinning model. Séminaire de probabilités.

[36] J. Poisat. Ruelle-perron-frobenius operator approach to the annealed pinning model with gaussian long-range disorder. Markov Proc. Relat. Fields, 19(3):577-606, 2013.

[37] D. Poland and H. A. Schegara. Occurence of a phase transition in nucleic acid models. J. Chem. Phys, 45:1464-1469, 1966.

[38] J. Sohier. Finite-size scaling for homogeneous pinning model. ALEA, 6:163-177, 2009.

[39] F. L. Toninelli. Critical properties and finite-size estimates for the depinning transition of directed random polymers. J. Stat. Phys., 126:1025-1044, 2007.

[40] F. L. Toninelli. Disordered pinning models and copolymers: beyond annealed bounds. Ann. Appl. Probab., 18:1569-1587, 2008.

[41] F. L. Toninelli. A replica-coupling approach to disordered pinning models. Commun. Math. Phys., 280:389-401, 2008.

[42] T. Vojta. Rare region effects at classical, quantum and nonequilibrium phase transitions. J. Phys. A.: Math. Gen., 39:143-205, 2006.

[43] A. Weinrib and B. I. Halperin. Critical phenomena in systems with long-range-correlated quenched disorder. Phys. Rev. B, $27: 413-427,1983$. 